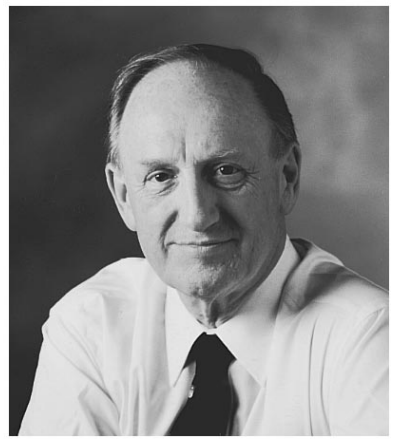

\section{Reading, Responding, and Recognition}

My 3 R's for this editorial are "Reading," "Responding," and "Recognition." At the recent SPIE meeting in San Diego I was asked how many people read any given paper in any given mainline journal. As far as I know, there have been no definitive studies of this question and hence it was a question to which I certainly didn't know the answer. My questioner persisted, however, and rephrased the question to "what did I think the range of responses might be to such a question?' I'm not exactly sure of my precise response, but it was something like "from less than ten to several hundred."' The "interviewer's" concern was that on average not many people really read any given paper. But is that important even if true? Is it the correct question? My conclusion is that the answer to the question is probably not of any great importance and is eventually misleading. Thus it is the wrong question!

What readers get from a journal article is some new knowledge or new point of view; much of that is gained by browsing rather than detailed study. This usually occurs just after the journal arrives on the reader's desk. The browsing results in considerable cross-fertilization of ideas from various sections of our field of optical science and engineering. It is precisely this cross-fertilization of ideas that makes me a champion of the broad coverage of journals such as Optical Engineering, as opposed to those that have a narrower and more specialized focus. Needless to say, these specialized journals have their place in the literature.

As the reader browses, other information gets stored in the reader's memory, i.e., some particular experimental or theoretical result that may not be of immediate importance, but may become important at a later time-and then the reader knows exactly where to go. Many R\&D groups have "journal sessions" as "periodical clubs", that meet on a regular basis to collectively look at recent papers of specific interest to the group. Often "readers" are assigned to particular journals and report back to the group. (How would you count the group members as readers?)

Original contributions to the literature inform the community of progress-progress that has at least the author- ity of the authors and the reviewers. Each contribution is like a piece of a jigsaw puzzle whose effect is multiplied by the presence of adjacent pieces, and then other pieces can be put in place, and so the forefront of science, engineering, and technology moves along (and often sideways to establish new parts of the puzzle to be solved). It is the integration of knowledge that allows us to see the "picture"' just as in a jigsaw puzzle. Often a group of isolated pieces of information don't fit in with the mainstream and must wait for the connection to be made. (The analogy to the jigsaw puzzle is particularly pertinent here.)

How often after worrying about a particular problem have you suddenly said (or thought), "Oh, I remember (blank) did something related to the question several years ago that may help us"...so back to finding and reading that journal article. The readers respond by integrating the new insights of a particular paper into their own thinking and future work. This provides recognition to the authors of the original work in a most satisfying way. Having your work positively referenced in future publications is certainly satisfying and does indeed provide public recognition. The citation indexes are measures of this recognition of an individual's contributions.

In another part of my conversation with my "interviewer' he made the very important comment that the real impact of a particular paper may not really be known until many years later. True indeed.

My conclusion is that refereed journals are the major source of authenticated progress in our field. So read, respond, and give recognition where recognition is due.

\section{Editor's Anecdote}

Some miscellaneous items from my mail:

"I apologize for the delay in studying the revised version. My printer broke.",

"I wonder how you ever found my current work address?',

"Because you are a famous scientist and there is a thick scientific atmosphere in your research group, I think my original ideals will be appreciated and my potential will be brought into full play.",

Brian J. Thompson Editor 